\title{
Changing negative core beliefs with trial-based thought record
}

\author{
Thaís R. Delavechia1, Michella L. Velasquez', Érica P. Duran², Lina S. Matsumoto², Irismar Reis de Oliveira ${ }^{1}$
}

${ }^{1}$ Department of Neurosciences and Mental Health, Postgraduate Programs (Medicine and Health, and Interactions of Organs and Systems), Federal University of Bahia (UFBA), Salvador, BA, Brazil. 2 Institute and Department of Psychiatry, University of São Paulo School of Medicine (IPq-HCFMUSP), São Paulo, SP, Brazil.

Received: $11 / 28 / 2015$ - Accepted: $4 / 6 / 2016$

DOI: 10.1590/0101-60830000000078

\begin{abstract}
Background: Trial-based thought record (TBTR) is a technique used in trial-based cognitive therapy (TBCT), and simulates a court trial. It was designed to restructure unhelpful core beliefs (CBs) during psychotherapy. Objective: To confirm previous findings on the efficacy of TBTR in decreasing patients' adherence to self-critical and unhelpful CBs and corresponding emotions, as well as assessing the differential efficacy of the empty-chair approach relative to the static format of TBTR. Methods: Thirty-nine outpatients were submitted to a 50-minute, one-session, application of the TBTR technique in the emptychair $(n=18)$ or conventional $(n=21)$ formats. Patients' adherence to unhelpful CBs and the intensity of corresponding emotions were assessed after each step of TBTR, and the results obtained in each format were compared. Results: Significant reductions in percent values both in the credit given to CBs and in the intensity of corresponding emotions were observed at the end of the session $(\mathrm{p}<.001)$, relative to baseline values. ANCOVA also showed a significant difference in favor of the empty-chair format for both belief credit and emotion intensity $(\mathrm{p}=.04)$. Discussion: TBTR may help patients reduce adherence to unhelpful CBs and corresponding emotions and the empty-chair format seems to be more efficacious than the conventional format.
\end{abstract}

Delavechia TR et al. / Arch Clin Psychiatry. 2016;43(2):31-3

Keywords: Cognitive therapy, core belief, Kafka, trial-based thought record, trial-based cognitive therapy.

\section{Introduction}

Trial-based cognitive therapy (TBCT) is a three-level, three-phase, transdiagnostic, case formulation approach, based on Aaron Beck's Cognitive Therapy, and inspired by the work of Franz Kafka. Joseph K., the main character in Kafka's novel, "The Trial"1, was unaware of the crime for which he was arrested and condemned without a chance to defend himself. According to de Oliveira's interpretation, a similar trial can be observed in patients seeking psychotherapy, who are constantly accusing and sentencing themselves through their negative core beliefs (CBs), but are unaware of such self-accusations and unable to organize their own self defense ${ }^{2}$.

Modifying dysfunctional CBs, understood as persistent attributions that are global, negative and personal regarding life events that are potential risk factors for the recurrence of most psychiatric disorders, often plays a significant role in cognitive behavioral therapy (CBT) protocols ${ }^{3}$. These beliefs are usually developed early in life and set the scene for later beliefs, assumptions and automatic thoughts. Once patients rarely question the validity of CBs, the process of accessing and modifying them, leading to a more constructive perception of current and future events, along with more enduring treatment outcomes, has become a frequent challenge and long-term goal for CBT therapists 4 .

Over the last decade, TBCT has helped several patients around the world become aware of their CBs (self-accusations) and develop more realistic and functional views of themselves ${ }^{5}$ as a result of its unique approach to conceptualization and techniques that make it a distinct intervention in modifying patients' $\mathrm{CBs}^{6}$. One of its main techniques is the trial-based thought record (TBTR), an intervention aimed at modifying dysfunctional CBs by simulating a legal trial. TBTR incorporates elements from various techniques already known in standard CBT and other psychotherapeutic approaches? The patient has the opportunity to role-play him/herself not only as the defendant, but also as the prosecutor and the defense attorney, as well as a member of the jury, as opposed to what usually happens when dysfunctional CBs are activated ${ }^{5}$. TBTR may be conducted using the empty chair format, an experiential approach derived from Gestalt Therapy, in which the patient moves to different chairs to role-play different characters, or in the static format, in which the patient remains in the same chair during the session.

The first study on the efficacy of the TBTR in changing dysfunctional CBs and their corresponding emotions was conducted ${ }^{7}$, and significant results $(\mathrm{p}<0.001)$ were observed in the adherence to CBs and associated emotions of 30 patients, reinforcing the hypothesis that this technique could, at least temporarily, contribute to the weakening of unhealthy CBs and their related emotions. Subsequently, the intensity of patients' dysfunctional CBs and corresponding emotions after the first use of the TBTR was assessed in a larger sample $(n=$ $166)^{8}$, confirming the previous findings.

In a randomized trial in which the TBTR was compared with traditional CBT strategies, 36 patients presenting a diagnosis of social anxiety disorder (SAD) were evaluated ${ }^{9}$. TBTR was at least as effective as conventional CBT in the treatment of SAD, and particularly effective in decreasing the fear of negative evaluation scores, supporting the relevance of further research with larger samples, and different disorders and populations. The same intervention also provided data demonstrating that TBTR was at least as effective as conventional CBT in improving several domains of quality of life in DAD. In addition, a significant treatment effect on the role-emotional domain at 12-month follow-up denoted a sustained effect of TBTR relative to conventional CBT10.

Given this context, the present study, besides aiming at confirming previous findings on the efficacy of TBTR in decreasing patients' adherence to their self-critical negative $\mathrm{CBs}$ and corresponding emotions, most importantly aimed at assessing the differential efficacy of the empty chair format of the TBTR, relative to the static format.

\section{Methods}

In this multi-center, parallel-group study, 39 outpatients presenting any psychiatric diagnoses were submitted to a 50-minute, onesession, application of the TBTR technique in the empty chair or static format by previously trained therapists, in the course of their CBT treatment.

A free web-based service that offers random assignment was used for randomization. A list of even/odd numbers (simple randomiza- 
tion) was used to separate the group treatments. As soon as a therapist from seven different capitals in Brazil decided to use the TBTR with a patient, he/she sent an email to the last author's secretary to inform the patient's initials and request the treatment modality (static versus empty chair) that should be used.

Patients' adherence to negative CBs and corresponding emotions were assessed after each step of the TBTR (investigation, prosecutor's plea, defense attorney's plea, prosecutor's second plea, defense attorney's second plea, jury's verdict and preparation for the appeal), and the results obtained in each format were compared. Statistical analyses involved a mixed (repeated measures) analysis of variance (ANOVA) to evaluate the efficacy of interventions. A one-way analysis of covariance (ANCOVA) was also conducted, using the baseline values from the investigation step of the TBTR as covariates. This research was approved by the Ethics Committee of Federal University of Bahia (Maternidade Climério de Oliveira), and all participants received detailed information about the study and signed an informed consent form.

\section{Results}

Results of the mixed ANOVA indicated a significant main effect, meaning that significant reductions in percent values both in the credit given to the $\mathrm{CBs}$ and in the intensity of the emotions were observed at the end of the session $(\mathrm{p}<.001)$, relative to baseline (investigation phase) values (Table 1). There was no significant interaction between time and treatment. However, ANCOVA showed a significant difference in favor of the empty chair approach not only in the percentage adherence to the negative CBs but also in the intensity of the corresponding emotions $(\mathrm{p}=.04)$.

\section{Discussion}

An intentional focus on changing CBs during the course of therapy has been widely emphasized in traditional CBT protocols ${ }^{4}$. However, as far as we know, little is understood about how cognitive change leads to symptom reduction ${ }^{11}$. Also, according to McManus et al. ${ }^{12}$ little is known about the efficacy of individual components of CBT, reinforcing the relevance of further understanding critical elements responsible for the efficacy, or comparing the efficacy of the different components in CBT protocols. Thus, in the present study, the authors aimed at supporting previous data on the efficacy of TBTR in reducing the strength of dysfunctional CBs as well as their associated emotions, not only in the static but also in the empty chair format of the intervention.

Similarly to what had previously been observed, significant reductions in the intensity of both negative unhelpful CBs and corresponding emotions were found at the end of the session, relative to initial values, as well as a significant difference in favor of the empty chair format for both belief credit and emotion intensity. The empty chair format has the advantage of engaging the patient in experiential learning, which is expected to be more effective in terms of belief and symptom change than other interventions that don't reach the gut level ${ }^{12,13}$, where enduring change is more likely to occur.

However, once TBTR incorporates several well-known techniques of conventional CBT and other psychotherapy approaches in its user-friendly structure, it can be quite challenging for the inexperienced clinician without proper training and supervision. Besides, addressing patients' unhelpful negative $\mathrm{CBs}$ often requires extra caution from therapists, who are expected to be truly empathic and respectful regarding their patients' experiences ${ }^{14}$.

Taking the above considerations into account, the TBTR may help patients reduce their attachment to negative CBs and corresponding emotions, confirming findings from previous studies, and the empty chair format seems to be more efficacious than the conventional format in reducing the intensity of associated emotions.

This study is limited by the short duration of observation (just one intervention). However, this was the only way to proceed, because TBTR is not necessarily repeated in subsequent sessions, precluding any additional comparison. Furthermore, the sample is heterogeneous, comprising patients with many different diagnoses. These are important aspects to be investigated in future studies with more homogeneous samples in order to determine the precise role

Table 1. Mean (SD) percentages of credit in the CBs and intensity of corresponding emotions in the total sample and in groups treated in the empty chair vs. static formats of TBTR

\begin{tabular}{|c|c|c|c|}
\hline TBTR steps & $\begin{array}{c}\text { Total sample } \\
\mathrm{n}=39\end{array}$ & $\begin{array}{c}\text { Empty chair } \\
n=18\end{array}$ & $\begin{array}{l}\text { Static } \\
n=21\end{array}$ \\
\hline $\begin{array}{l}\text { 1. Investigation } \\
\text { Belief } \\
\text { Emotion }\end{array}$ & $\begin{array}{l}85.13(19.21) \\
82.18 \text { (19.99) }\end{array}$ & $\begin{array}{l}82.50 \text { (22.90) } \\
79.72 \text { (19.59) } \\
\end{array}$ & $\begin{array}{l}87.38(16.62) \\
84.29(20.57)\end{array}$ \\
\hline $\begin{array}{l}\text { 2. Prosecutor I } \\
\text { Belief } \\
\text { Emotion }\end{array}$ & $\begin{array}{l}91.15(14.49) \\
89.87(14.44)\end{array}$ & $\begin{array}{l}92.78(10.74) \\
92.78(11.27) \\
\end{array}$ & $\begin{array}{l}89.76 \text { (17.21) } \\
87.38 \text { (16.55) }\end{array}$ \\
\hline $\begin{array}{l}\text { 3. Defense attorney I } \\
\text { Belief } \\
\text { Emotion }\end{array}$ & $\begin{array}{l}56.54(20.30) \\
54.36(22.98)\end{array}$ & $\begin{array}{l}57.78(17.00) \\
54.72(21.04)\end{array}$ & $\begin{array}{l}55.48(23.12) \\
54.05(25.03) \\
\end{array}$ \\
\hline $\begin{array}{l}\text { 4. Prosecutor II } \\
\text { Belief } \\
\text { Emotion }\end{array}$ & $\begin{array}{l}74.10 \text { (24.71) } \\
70.51 \text { (25.59) }\end{array}$ & $\begin{array}{l}73.33(25.44) \\
72.78(26.08)\end{array}$ & $\begin{array}{l}74.76(24.67) \\
68.57(25.65)\end{array}$ \\
\hline $\begin{array}{l}\text { 5. Defense attorney II } \\
\text { Belief } \\
\text { Emotion }\end{array}$ & $\begin{array}{l}39.62(18.22) \\
35.80(21.83)\end{array}$ & $\begin{array}{l}38.33(18.55) \\
32.50(19.42) \\
\end{array}$ & $\begin{array}{l}40.71(18.32) \\
38.62(23.80) \\
\end{array}$ \\
\hline $\begin{array}{l}\text { 6. Jury's verdict } \\
\text { Belief } \\
\text { Emotion }\end{array}$ & $\begin{array}{l}25.90(19.26) \\
22.41(19.72) \\
\end{array}$ & $\begin{array}{l}20.00(15.62) \\
16.11(13.78) \\
\end{array}$ & $\begin{array}{l}30.95(20.95) \\
27.81(22.60)\end{array}$ \\
\hline $\begin{array}{l}\text { 7. Appeal preparation } \\
\text { Belief } \\
\text { Emotion }\end{array}$ & $\begin{array}{l}18.08(15.42) \\
16.20(14.70)\end{array}$ & $\begin{array}{l}12.22(10.46) \\
10.83(10.88) \\
\end{array}$ & $\begin{array}{l}23.10(17.35) \\
20.81(16.18)\end{array}$ \\
\hline $\begin{array}{l}\text { Improvement } \\
\text { Belief } \\
\text { Emotion }\end{array}$ & $\begin{array}{l}76.24(21.81) \\
79.10(18.86)\end{array}$ & $\begin{array}{l}79.68(24.19) \\
83.45(19.55)\end{array}$ & $\begin{array}{l}73.30 \text { (19.66) } \\
75.38(17.87)\end{array}$ \\
\hline
\end{tabular}


of TBTR in helping patients to modify their negative CBs and to reduce self-criticism.

\section{Conflicts of interest}

The authors do not report any conflicts of interest. Trial-based cognitive therapy was developed by one of the authors (IRO). This research was not funded.

\section{References}

1. Kafka F. The Trial. New York: Schoken Books, 1998.

2. De Oliveira IR. Kafka's trial dilemma: proposal of a practical solution to Joseph K's unknown accusation. Med Hypotheses. 2011;77(1):5-6.

3. Beck JS. Cognitive behavior therapy: basics and beyond. 2ed rev. New York: Guilford Press, 2011.

4. Wenzel A. Modification of core beliefs in cognitive therapy. In: De Oliveira IR (Ed.). Standard and Innovative Strategies in Cognitive Behavior Therapy. 2012. InTech. Available from: http://www.intechopen. com/books/standard-and-innovative-strategies-in-cognitive-behaviortherapy.

5. De Oliveira IR. Trial-based cognitive therapy: a manual for clinicians. New York: Routledge, 2015.

6. de Oliveira IR. Trial-based cognitive therapy (TBCT): a new cognitivebehavior therapy approach. In: De-Oliveira IR, Scwartz T, Stahl SM (eds.). Integrating psychotherapy and psychopharmacology: A handbook for clinicians. New York: Routledge, 2014.
7. de Oliveira IR. Trial-based thought record (TBTR): preliminary data on a strategy to deal with core beliefs by combining sentence reversion and the use of an analogy to a trial. Rev Bras Psiquiatr. 2008;30(1):12-8.

8. de Oliveira IR, Hemmany C, Powell VB, Bonfim TD, Duran EP, Novais N, et al. Brazilian TBTR Study Group. Trial-based psychotherapy and the efficacy of trial-based thought record in changing unhelpful core beliefs and reducing self-criticism. CNS Spectr. 2012;17(1):16-23.

9. de Oliveira IR, Powell VB, Wenzel A, Caldas M, Seixas C, Almeida C, et al. Efficacy of the trial-based thought record, a new cognitive therapy strategy designed to change core beliefs, in social phobia. J Clin Pharm Ther. 2012;37(3):328-34

10. Powell VB, de Oliveira OH, Seixas C, Almeida C, Grangeon MC, Caldas $\mathrm{M}$, et al. Changing core beliefs with trial-based cognitive therapy my improve quality of life in social phobia: a randomized study. Rev Bras Psiquiatr. 2013;35(3):243-47.

11. Longmore RJ, Worrell M. Do we need to challenge thoughts in cognitive behavior therapy? Clin Psychol Rev. 2007;27(2):173-87.

12. McManus F, Van Doorn K, Yiend J. Examining the effects of thought records and behavioral experiments in instigating belief change. J Behav Ther Exp Psychiatry. 2012;43(1):540-7.

13. Rouf K, Fennell MJV, Westbrook D, Cooper M, Bennett-Levy J. Devising effective behavioural experiments. In: Bennett-Levy J, Butler G, Fennell MJV, Hackmann A, Mueller M, Westbrook D (eds.). Oxford guide to behavioural experiments in cognitive therapy. Oxford: Oxford University Press, 2004.

14. James IA, Barton S. Changing core beliefs with the continuum technique. Behav Cogn Psychother. 2004;(32):431-42. 